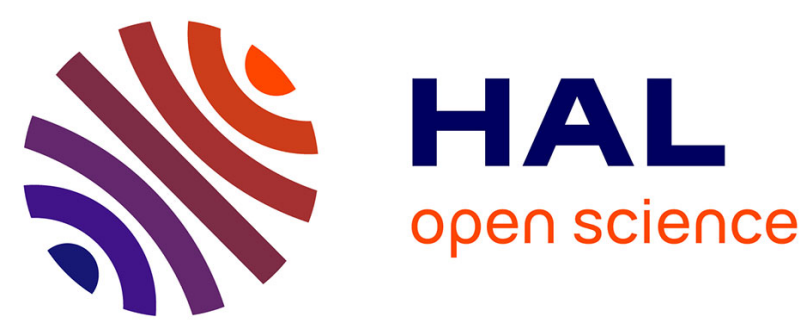

\title{
Picosecond Short-Range Disordering in Isochorically Heated Aluminum at Solid Density
}

\author{
A. Mancic, A. Levy, M. Harmand, M. Nakatsutsumi, P. Antici, P. Audebert, \\ P. Combis, S. Fourmaux, S. Mazevet, O. Peyrusse, et al.
}

\section{- To cite this version:}

A. Mancic, A. Levy, M. Harmand, M. Nakatsutsumi, P. Antici, et al.. Picosecond Short-Range Disordering in Isochorically Heated Aluminum at Solid Density. Physical Review Letters, 2010, 104 (3), 10.1103/PhysRevLett.104.035002 . hal-01561864

\section{HAL Id: hal-01561864 https://hal.science/hal-01561864}

Submitted on 30 Mar 2021

HAL is a multi-disciplinary open access archive for the deposit and dissemination of scientific research documents, whether they are published or not. The documents may come from teaching and research institutions in France or abroad, or from public or private research centers.
L'archive ouverte pluridisciplinaire HAL, est destinée au dépôt et à la diffusion de documents scientifiques de niveau recherche, publiés ou non, émanant des établissements d'enseignement et de recherche français ou étrangers, des laboratoires publics ou privés.

\section{(c)(1)}

Distributed under a Creative Commons Attribution| 4.0 International License 


\title{
Picosecond Short-Range Disordering in Isochorically Heated Aluminum at Solid Density
}

\author{
A. Mančić, ${ }^{1}$ A. Lévy, ${ }^{2}$ M. Harmand, ${ }^{2}$ M. Nakatsutsumi, ${ }^{1}$ P. Antici,,${ }^{3,4}$ P. Audebert, ${ }^{1}$ P. Combis,${ }^{5}$ S. Fourmaux, ${ }^{6}$ S. Mazevet, ${ }^{5}$ \\ O. Peyrusse, ${ }^{2}$ V. Recoules, ${ }^{5}$ P. Renaudin, ${ }^{5}$ J. Robiche, ${ }^{1, *}$ F. Dorchies, ${ }^{2}$ and J. Fuchs ${ }^{1, \dagger}$ \\ ${ }^{1}$ LULI, École Polytechnique, CNRS, CEA, UPMC, route de Saclay, 91128 Palaiseau, France \\ ${ }^{2}$ Université de Bordeaux-CNRS-CEA, Centre Lasers Intenses et Applications (CELIA), Talence, F-33405, France \\ ${ }^{3} I L E$-École Polytechnique-CNRS-ENSTA-Iogs-UP Sud, Batterie de l'Yvette, 91761 Palaiseau, France \\ ${ }^{4}$ Istituto Nazionale di Fisica Nucleare, Via E. Fermi, 40-00044 Frascati, Italy \\ ${ }^{5} C E A, D A M, D I F$, F-91297 Arpajon, France \\ ${ }^{6} I N R S$-Energie et Matériaux, 1650 BD. L. Boulet, J3X1S2 Varennes, Québec, Canada
}

(Received 5 October 2009; published 20 January 2010)

\begin{abstract}
Using ultrafast x-ray probing, we experimentally observed a progressive loss of ordering within soliddensity aluminum as the temperature raises from $300 \mathrm{~K}$ to $>10^{4} \mathrm{~K}$. The $\mathrm{Al}$ sample was isochorically heated by a short $(\sim \mathrm{ps})$, laser-accelerated proton beam and probed by a short broadband $\mathrm{x}$-ray source around the $\mathrm{Al} K$ edge. The loss of short-range ordering is detected through the progressive smoothing of the time-resolved x-ray absorption near-edge spectroscopy (XANES) structure. The results are compared with two different theoretical models of warm dense matter and allow us to put an upper bound on the onset of ion lattice disorder within the heated solid-density medium of $\sim 10 \mathrm{ps}$.
\end{abstract}

DOI: 10.1103/PhysRevLett.104.035002

Understanding the properties of the warm dense matter (WDM) regime and of the phase transitions leading to this regime [1] is of considerable interest since it is found in a large array of conditions, e.g., astrophysical objects or controlled thermonuclear fusion compressions. Initial experiments on WDM states used mostly reflectivity [2] or conductivity $[3,4]$ as observables. These experiments gave valuable data points to be compared with theory but they did not yield direct access into the time evolution of the structural properties as the system transits from a solid to a WDM state.

Detailed structural information can be extracted, e.g., from the time evolution of diffraction patterns, using either electron or $\mathrm{x}$ ray [5], as they reflect the underlying crystallographic structures. However, once the system has melted, a diffraction pattern is no longer present and very little is currently known about the structural evolution towards the plasma state. Note that angularly resolved x-ray scattering [6] was also proposed to get snapshots of changing ionic and electronic structures in WDM but was only applied to slowly (ns) shock-compressed materials. We should mention as well the time-resolved x-ray diffraction studies of ultrafast structural changes in materials performed on synchrotrons [7], where it was shown that $\mathrm{x}$-ray pulses are a suitable tool for the study of the temporal dynamics of condensed matter phase transitions. However, those works were primarily focused on surface disordering of thin films or of surface layers.

Here, we study the structural modification of thick samples of solid-density aluminum heated up to a few $\mathrm{eV}$ at the ps time scale. For this, we exploit two techniques that allow us to uniquely create solid-density, $\mu \mathrm{m}$ thick matter heated in the $\mathrm{eV}$ range and to probe its ionic structure in a
PACS numbers: 52.27.Gr, 32.30.Rj, 52.25.Os, 52.50.Gj

time-resolved fashion. First, as shown in Fig. 1(a), a shortburst $(\sim \mathrm{ps})$, laser-accelerated proton beam is used to heat isochorically and uniformly a thick $(\mu \mathrm{m}) \mathrm{Al}$ sample over $\sim 25$ ps, i.e., shorter than the hydrodynamic expansion time, as detailed in Ref. [8]. This allows us to keep soliddensity conditions over $60 \mathrm{ps}$ after the beginning of heat-

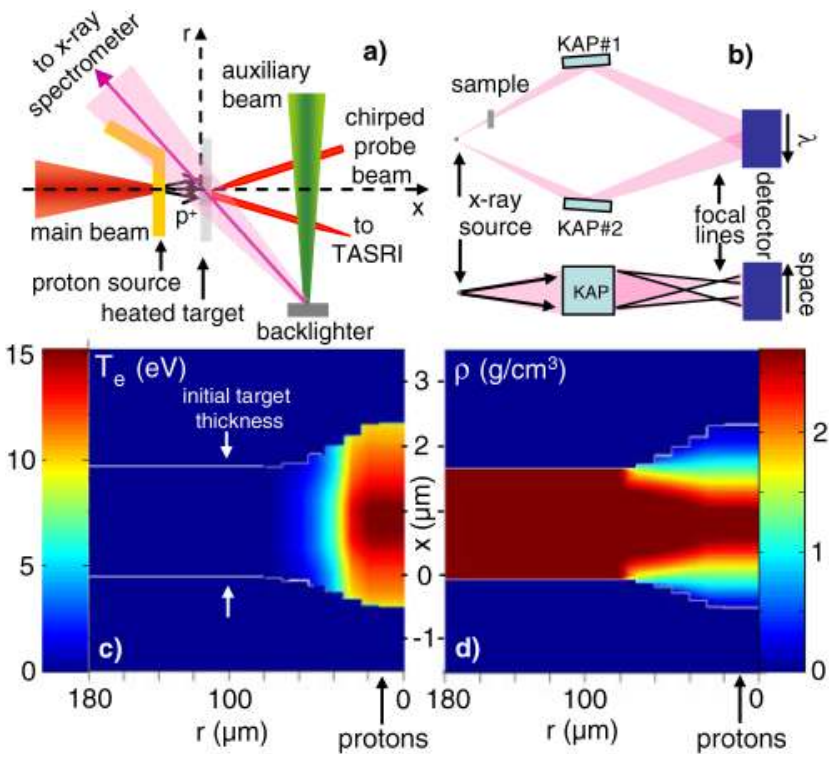

FIG. 1 (color online). (a) Setup of the experiment-top view; (b) schematic representation of the $\mathrm{x}$-ray spectrometer; (c) temperature profile (in $\mathrm{eV}$ ) of the heated $1.6 \mu \mathrm{m}$ thick $\mathrm{Al}$ foil $45 \mathrm{ps}$ after the first protons have left the source target as given by self-consistent simulations. The $x$ axis corresponds to the axis of heating while the other axis corresponds to the radial dimension; (d) density profile (in $\mathrm{g} / \mathrm{cm}^{3}$ ) of the same target at the same moment. 
ing. Second, we use an ultrafast broadband x-ray probe beam [9] to perform time-resolved x-ray absorption nearedge spectroscopy (XANES) [10]. The XANES absorption spectrum is very sensitive to the local atomic arrangement and can provide direct insight into the structural properties of matter. X-ray photoabsorption was previously used in slow (ns) shock-compression experiments [11,12] and calculations [13] but the main focus was on the position and the width of the $K$ edge as the density and temperature increased. However, the full structure of the spectrum was not exploited to get structural information about the phase transition.

Experimentally, we observe the smoothing of the XANES structure of solid aluminum as it is isochorically heated from room temperature to several eVs. The observations are correlated to quantum molecular dynamic (QMD) calculations as well as a simpler liquid description [14-17], and good agreement, qualitative as well as quantitative, is found. Such comparison allows conclusively to relate the observed XANES spectral modifications to structural disordering of the heated material. We can thus, for the first time put an upper limit of $\sim 10$ ps on the loss of short-range ordering during this solid-to-plasma transition.

The experiment was performed using the LULI $100 \mathrm{TW}$ laser facility working in the chirped pulse amplification mode at a wavelength $1.057 \mu \mathrm{m}$. The experimental setup is shown in Fig. 1(a). The main laser pulse compressed to 320 fs full width at half maximum (FWHM) irradiated a $10 \mu \mathrm{m}$ thick Au flat foil (the "proton source" target) at normal incidence with a peak intensity of $\sim 3 \times$ $10^{19} \mathrm{~W} / \mathrm{cm}^{2}$, creating a pulsed, highly directional, multi$\mathrm{MeV}$ proton beam [18]. This beam induced the isochoric heating of a flat Al foil ("heated" target), which was positioned $200 \mu \mathrm{m}$ away from the proton source target [8]. A single-shot time- and space- resolved interferometry (TASRI) diagnostic system [19] monitored the phase of a chirped probe beam $(1.057 \mu \mathrm{m})$ of $50 \mathrm{ps}$ duration, reflecting at a $15^{\circ}$ incidence on the rear surface of the protonheated target [8]. It allows us to measure the expansion velocity of the heated target critical density interface.

An ultrashort broadband x-ray pulse was used to backlight the heated sample. It was created with a synchronized, $5 \mathrm{~J}$ energy, 1.4 ps duration, $0.53 \mu \mathrm{m}$ wavelength beam focused on an Er target. In a previous study, optimization of the x-ray source around the $\mathrm{Al} K$ edge (1.50 to $1.75 \mathrm{keV}$ ) was achieved [20,21]. Erbium was chosen for emitting preferentially in the spectral range covering the Al $K$ edge. The x-ray spectra (integrated over the duration of the $\mathrm{x}$-ray probe that is $\sim 4$ ps [22]) were recorded with a specific x-ray absorption spectrometer composed of two KAP diffractive conical crystals [see Fig. 1(b)]. The first one collected directly the $\mathrm{x}$-ray source emission while the other one looked through the heated $\mathrm{Al}$ sample, measuring the transmitted spectrum. This configuration allowed simultaneous measurement of the transmitted and reference spectra, yielding the transmission $T_{r}$ from their ratio. The absorption spectrum $A$ was deduced from the relation $T_{r}=$ $\exp (-A d)$, where $d$ is the thickness of the Al sample. In this way, the influence of shot-to-shot fluctuations of the $\mathrm{x}$-ray source on the determination of $A$ was eliminated. An image plate was used as X-ray detector [23]. As shown in Fig. 1(b), it was placed after the focal line of the crystals. This enables, in the dimension transverse to the spectral dispersion axis, spatial resolution (by point-projection) in the heated sample. Spatial and spectral resolutions were, respectively, $28 \mu \mathrm{m}$ and $1.9 \mathrm{eV}$. The thickness of the $\mathrm{Al}$ sample $(d=0.5$ or $1.6 \mu \mathrm{m})$ was chosen to optimize the signal-to-noise ratio in the absorption spectra. Experimental constraints required averaging the $\mathrm{x}$-ray data radially over $170 \mu \mathrm{m}$ in the heated target, starting from the axis of the proton beam irradiation (with an error of $\pm 30 \mu \mathrm{m})$.

The heated target conditions at different times during heating are inferred by coupling [8] the TASRI diagnostic with the hydrodynamic code ESTHER [24]. This procedure shows that heating was isochoric and almost uniform along the target thickness (axis $x$ ). However, in the radial dimension (axis $r$ ) heating is not uniform over the $170 \mu \mathrm{m}$ probed area. The radius of the heated surface is limited by the maximum divergence angle of the proton beam and is estimated to be $\sim 80 \mu \mathrm{m}$. This agrees well with complementary 2D time-resolved measurements of the rear surface emissivity of the heated target [8]. An example of such inferred density $(\rho)$ and temperature $(T)$ conditions for a $1.6 \mu \mathrm{m}$ thick Al target is shown in Figs. 1(c) and 1(d). These are obtained 45 ps after the protons have left the source, illustrating that most of the target density stays solid (except at the edges) over a relatively long duration. The simulation assumes electron-ion equilibrium, i. e., $T_{e}=T_{i}=T$. The radial variation of $T$ implies that the plasma conditions will be averaged over the radial range of $\mathrm{x}$-ray probing. A maximum temperature of $15 \mathrm{eV}$ at solid density could be reached, however, the mean temperature integrated over the radial dimension of the heated area is in the $\mathrm{eV}$ range. Controlling the time delay between the two laser beams (proton source and x-ray probe) allows us to probe the sample in different states. In addition, the heating conditions may be varied by altering the thickness of the Al sample or modulating the proton source by defocusing the main laser beam. By changing these parameters, a set of $K$ edge absorption spectra was obtained. Some results are presented in Fig. 2(a) with the corresponding inferred average temperature (detailed below). The "cold" spectrum corresponds to a shot without a proton beam. This shows the XANES modulations for $\mathrm{x}$-ray photon energies above the $K$ edge.

For heated targets, one of the features that can be clearly observed is the decrease of the slope around the $K$-absorption edge ( $K$ edge broadening), with this decrease becoming more pronounced as $T$ increases. Another obvious feature is the progressive smoothing of the absorption spectra just after the $K$ edge (i.e., for $E>1.56 \mathrm{keV}$ ) with a rising temperature. This corresponds to the disappearance of the 1 st and 2 nd bumps (indicated by the 

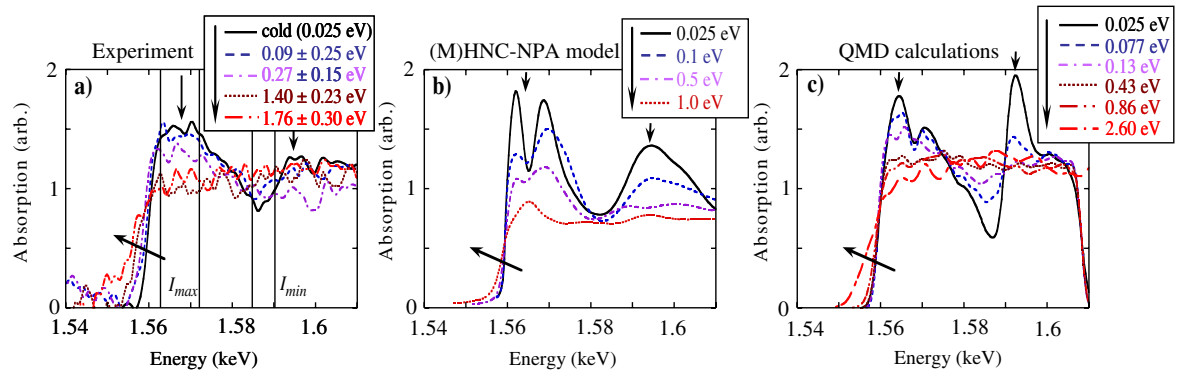

FIG. 2 (color online). (a) Experimentally measured XANES spectra near the $K$ edge. The spectra are normalized to the mean value without the XANES structure. The "cold shot" corresponds to a shot without heating proton beam; (b), (c) Calculated $K$ edge XANES spectra (normalized to the mean value without XANES structures) of aluminum as a function of the temperature (solid density): (b) (M)HNC-NPA model, (c) QMD data.

vertical arrows in Fig. 2) of the XANES modulations that transform in a broad plateau for $T>1 \mathrm{eV}$. This effect is related to a structural modification of the heated sample. Indeed, according to XANES theory [25], the modulations observed in the spectrum depend on the local geometry of the atoms involved in the x-ray absorption transition, i.e., they are directly related to the short-range order. As a consequence, the observed smoothing of the XANES structure can be attributed to a progressive loss of ion-ion order in the produced isochoric WDM.

The averaged temperatures of the sample, $T$, given above, are estimated using a simple model of x-ray absorption based on Fermi-Dirac statistics. This model assumes a free electron gas behavior for the valence electrons and the absorption is taken to be proportional to the vacancy for the photoelectron, i. e., $\sim[1-f(E)]$, where $f(E)$ is the Fermi-Dirac distribution function. Using this formula, a one-to-one correspondence is found between the $K$ edge slope and the temperature $T$. Thus, the average temperature of the heated $\mathrm{Al}$ sample can be inferred from the measured spectra. This method is validated by a comparison with two theoretical models (see below). From such comparison, we observe that due to the simplified modeling of the absorption spectra, the temperature is estimated with an accuracy of $15 \%$. This, with the noise in the experimental data, results in the uncertainty in the estimated $T$ shown in Fig. 2(a). We have also checked that our average temperature retrieval process is consistent with that inferred from the ESTHER simulated target conditions. For this, we use the simulated $(\rho, T)$ to calculate, according to the same FermiDirac model, the absorption along the probing $\mathrm{x}$-ray lines passing through the target at different radial positions. Then, from the slope of the averaged $K$ edge profile, the average $T$ is estimated.

We turn toward the results of two theoretical models to get insight into the observed modifications of the XANES spectra with increasing temperature. The first one involves a (modified) hypernetted chain-neutral pseudo atom (MHNC-NPA) model of dense matter coupled with a specific finite difference modeling of XANES structures and is described in Refs. [14,15]. The second theoretical approach, described in Refs. [16,17], uses QMD simulations in the framework of density functional theory. This approach provides a consistent description of both the state of the plasma and the optical response in the x-ray domain. The absorption spectra calculated for different temperatures using the two different approaches are given in Figs. 2(b) and 2(c). Comparing Figs. 2(a)-2(c), both the experimental results and the calculations exhibit the same behavior: (i) a $K$ edge broadening with increasing temperature and (ii) a loss of structure in a similar range of temperature.

Both models assume electron-ion equilibrium. The evolution of the radial distribution function $g(r)$-which is related to the probability of finding another particle at a distance $r$ from a given particle-as a function of temperature (given in Ref. [14,17]) confirms that the smoothing of the XANES spectra corresponds to the loss of ordering in the sample. We point out, however, that while the disappearance of the XANES structure corresponds to a loss of ordering in the liquid, there remains some ordering in the system when the XANES spectra is flat [17]. Note also that Figs. 2(b) and 2(c) further show that while the two models predict that the XANES structure disappears, they do so for different temperatures. The MHNC-NPA shows some structure remaining at $T=1 \mathrm{eV}$ while the QMD simulations predict a structureless spectra for temperatures above $0.43 \mathrm{eV}$.
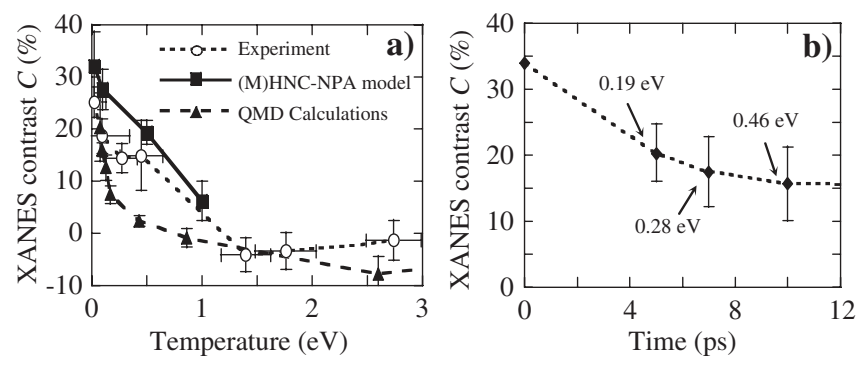

FIG. 3. Contrast $C(\%)$ of the XANES modulations (a) as a function of the temperature for experimental and theoretical data and (b) as a function of time after the first protons have left the proton source (the arrival of the first protons in the heated $\mathrm{Al}$ target starts at $t \sim 3 \mathrm{ps}$ ) with indicated average temperatures. 
To quantitatively compare experimental and calculated XANES profiles, we have calculated a "contrast" $C$ for each profile: $C(\%)=\left(I_{\max }-I_{\min }\right) /\left(I_{\max }+I_{\min }\right)$, where $I_{\max }$ and $I_{\min }$ are average values of the absorption signal around the first XANES maximum and minimum, respectively. The location of $I_{\max }$ and $I_{\min }$ is shown in Fig. 2(a). Results are reported as a function of temperature in Fig. 3(a). We can see that the evolution of both experimental and calculated contrasts is similar. For lower temperatures $(T<0.4 \mathrm{eV})$, QMD calculations seems to agree better with the measurements while for the higher temperatures the MHNC-NPA model agrees better.

We point out that QMD simulations have been rather successful at describing the electronic transport properties for aluminum in the solid, liquid, and plasma states and (as such) we expect that the structure of the system is well described in the density-temperature range explored here. On the other hand, there clearly remains a need to validate the impurity model, in which the absorbing electron is described by an "excited pseudopotential" by performing further measurements around $0.5 \mathrm{eV}$. In contrast, the approach based on MHNC-NPA is developed to model the warm dense regime and for (high) temperatures where it is computationally too expensive to apply QMD simulations. Independently of the precise finite difference treatment of the XANES spectrum itself, this approach still needs to be validated in the regime between solid and liquid that is studied here. The qualitative comparison with the experimental result in this regime is very promising and further comparison with the QMD results is needed to understand the differences between the two models and to improve each component of the MHNC-NPA model in the region where both QMD and the MHNC-NPA model can be applied.

To obtain information about the temporal dynamics of the observed ordering loss, we follow in time the simulated target conditions [as shown for one late time in Figs. 1(c) and 1(d)] and use them to compute averaged XANES spectra similarly as in the experiment. For this, we exploit the QMD spectra calculated at solid density for various temperatures. We checked that for each calculation, we could use these spectra as the electron-ion equilibrium was reached. This was done by solving the differential equations giving the energy transfer between electrons (that are heated collisionally by the incident protons) and the lattice ions, similarly as in Refs. [26,27]. Finally, we calculate the contrast of the simulated average spectrum at each time, as shown in Fig. 3(b). We observe that the contrast falls rapidly, in $10 \mathrm{ps,}$, while the average temperature increases. At this point, according to the theoretical models, the medium becomes structureless. We can therefore put an upper bound of $10 \mathrm{ps}$ on the loss of short-range ordering.

The techniques employed here can provide direct insight into the dynamic of the solid-to-plasma phase transition. Further experiments will be oriented toward enhancing the temporal resolution by shortening the duration of the proton heating and of the $\mathrm{x}$-ray probing as well as improving the spatial resolution of the XANES measurements so that the averaging, which was necessary here, may be minimized.

We acknowledge the expert support from the technical groups at LULI. This work was supported by the Grant No. E1127 from Région Ile-de-France, by the Ministry of Science, Serbia (Project No. 141034) and ANR-06-BLAN0392 from ANR-France. M. N. received partial support from JSPS.

*Present address: CEA IRAMIS Service des Photons, Atomes et Molecules, F-9191, Gif sur Yvette, France. †julien.fuchs@polytechnique.fr

[1] S. Ichimaru, Rev. Mod. Phys. 54, 1017 (1982); R. W. Lee et al., J. Opt. Soc. Am. B 20, 770 (2003).

[2] A. Ng et al., Phys. Rev. Lett. 72, 3351 (1994); A. N. Mostovych and Y. Chan, Phys. Rev. Lett. 79, 5094 (1997); H. Yoneda et al., Phys. Rev. Lett. 91, 075004 (2003); T. Ao et al., Phys. Rev. Lett. 96, 055001 (2006).

[3] H. Kunze and A. DeSilva, Phys. Rev. E 49, 4448 (1994).

[4] P. Renaudin et al., Phys. Rev. Lett. 88, 215001 (2002).

[5] R. Ernstorfer et al., Science 323, 1033 (2009).

[6] S.H. Glenzer et al., Phys. Plasmas 10, 2433 (2003); B. Barbrel et al., Phys. Rev. Lett. 102, 165004 (2009).

[7] J. Larsson et al., Appl. Phys. A 66, 587 (1998).

[8] A. Mancic et al., High Energy Density Phys. 6, 21 (2009).

[9] P. Audebert et al., Phys. Rev. Lett. 94, 025004 (2005).

[10] C. Bressler and M. Chergui, Chem. Rev. 104, 1781 (2004).

[11] B. K. Godwal et al., Phys. Rev. A 40, 4521 (1989); D. K. Bradley et al., Phys. Rev. Lett. 59, 2995 (1987).

[12] T. A. Hall et al., Europhys. Lett. 41, 495 (1998).

[13] F. Perrot and M. W. C. Dharma-wardana, Phys. Rev. Lett. 71, 797 (1993).

[14] O. Peyrusse, J. Phys. Condens. Matter 20, 195211 (2008).

[15] O. Peyrusse et al., in Atomic Processes in Plasmas, edited by K. Fournier, AIP Conf. Proc. No. 1161 (AIP, New York, 2009), p. 200.

[16] S. Mazevet and G. Zérah, Phys. Rev. Lett. 101, 155001 (2008).

[17] V. Recoules and S. Mazevet, Phys. Rev. B 80, 064110 (2009).

[18] J. Fuchs et al., C.R. Physique 10, 176 (2009).

[19] J. P. Geindre et al., Opt. Lett. 26, 1612 (2001).

[20] M. Harmand et al., Phys. Plasmas 16, 063301 (2009).

[21] F. Dorchies et al., Appl. Phys. Lett. 93, 121113 (2008).

[22] C. Chenais-Popovics et al., J. Phys. IV (France) 108, 137 (2003).

[23] Y. Amemiya, J. Synchrotron Radiat. 2, 13 (1995).

[24] J.P. Colombier et al., Phys. Rev. B 71, 165406 (2005).

[25] J. J. Rehr and R.C. Albers, Rev. Mod. Phys. 72, 621 (2000).

[26] A. Kemp et al., Phys. Rev. E 75, 056401 (2007).

[27] H. Chen and S. C. Wilks, Laser Part. Beams 23, 411 (2005). 